\title{
62 \\ A Model of Co-ordination Support for Unanticipated Situations *
}

\author{
S. Ossowski and A. García-Serrano \\ Department of Artificial Intelligence \\ Technical University of Madrid \\ Campus de Montegancedo s/n, Boadilla del Monte 28660, Spain \\ Phone: (+34-1) 352-48-03, Fax: (+34-1) 352-48-19 \\ E-mail: \{ossowski, agarcia\}@dia.fi.upm.es
}

\begin{abstract}
A mayor drawback of today's workflow management approaches is their difficulty in responding adequately to unexpected events. In this paper, we model a social mechanism from which dynamic workflow adaptation emerges: self-interested actors laterally co-ordinate their activities in order to restore a desired process functionality. Finally, the architecture of an agent-based support system that assists flexible exception management is sketched.
\end{abstract}

Keywords

Co-ordination, Intelligent Workflow Management, Computer-Supported Co-operative Work, Distributed Artificial Intelligence

\section{INTRODUCTION}

The effective management of business processes has become a decisive factor for the success of modern organisations. Workflow management systems (WFMS) address this ubiquitous task by rendering support to strategic and operational issues such as analysis, formal representation, simulation, monitoring, control and documentation of business processes.

In this paper we are concerned with the co-ordination aspects of workflow management: we are interested in rendering support to the articulation of work processes in order to foster the smooth flow of work within the organisation. This imposes requirements on the tasks and their relation within processes as well as on the actors that enact them: on the one hand a smooth flow of work needs to be composed of sequences of tasks which are intellectually manageable and operationally efficient (Nutt, 1993). On the other, it requires an even distribution of work among actors (Bowers et al., 1995).

Today's WFMS instantiate models of routine processes before enactment, making binding commitments concerning all relevant aspects of the constituent tasks. This instantiation implies an a priori co-ordination of process activities: it excludes all those process instances which are believed to be inefficient or unmanageable within a certain expected situation.

\footnotetext{
* This work was supported by the Human Capital and Mobility Program (HCM) of the European Union, contract ERBCHBICT941611, and by Spanish CDTI, project 01594-PC019 (BIOS)
} 
Such an approach is problematic for two reasons. Firstly, it is usually too prescriptive for human actors, as it "overspecifies" their work. The support system frequently imposes a new (presumably "better") way of doing things, which is alien to the actors' own methods of organising their work. Secondly, it is inflexible with respect to exception handling and "online" adaptation to changing circumstances, as it is often impossible to derive all relevant parameters of workflows a priori. Robinson (1993) stresses that no a priori workflow, no anticipated sequence of events will ever match the rich, concrete detail of an actual situation.

In recent years there are attempts to overcome the above drawbacks by providing process description with information concerning the context of tasks Contributions from the Database community focus on advanced transaction models: in case of a failure during process enactment, already committed tasks are successively "undone" until a consistent state is reached (Eder and Liebhart, 1996). Artificial Intelligence (AI) approaches provide knowledge-based reasoning mechanisms to manage dependencies between tasks (Malone and Crowston, 1994; Singh and Huhns 1995). However, decisions on the "route" of workflow recovery are usually modelled from the perspective of an external observer: they are delegated to a central "authority".

In this paper, we are interested in having the functionality of such a central authority emerge from the social context of the actors that perform the tasks. We model the process of re-coordination from the standpoint of an observer located within a group of actors: the shape of the adapted workflow arises from the individual decisions of self-interested actors, that comprise aspects of strategic decision-making and normative reasoning.

\section{Flow Processes and Exceptions}

Organisational processes can be modelled at two levels of abstraction: at the distribution level the way in which a process is performed is settled and its different tasks assigned to the actors' work lists. At the scheduling level temporal aspects of process enactment are taken into account: actors sequence the task in their work lists and synchronise them by assigning consistent execution times.

In the sequel we consider organisational processes at the distribution level and will not address the question of how actors organise their individual work. This leads to the notion of flow processes: a certain amount of input work items per time unit (input flow) is successively transformed into output work items (output flow). Such an abstraction is suitable for routine process, in the course of which big amounts of uniform work items are processed.

Definition 1: A simple flow process (SFP) is a triple $P_{S}=(G, A, \delta)$ where:

- $G=(T, F)$ is a directed acyclic graph. The set $T$ of nodes (tasks) is partitioned in three disjunctive subsets: work tasks (WT), routing tasks (RT) and co-operative task (CT) respectively. Arcs are described by the flow relation F $\subset$ TxT such that CTs and WTs have exactly one predecessor and one successor; RTs have either one successor or one predecessor. Tasks without predecessors are called start nodes, tasks without successors are end nodes.

- $\mathrm{A}$ is a set of actors.

- $\delta: T \rightarrow \wp(\mathrm{A})$ is a task assignment function where $|\delta(\mathrm{t})|=1$ if $t \in \mathrm{WT} \cup \mathrm{RT}$.

A SFP can be graphically depicted as a graph, where streams of uniform work items flow along arcs, i.e. arcs can be interpreted as producer-consumer dependencies between tasks. WTs and CTs are intended to model activities that transform work items, so they are endowed with only one predecessor and one successor task. WTs are performed by one actor while several actors may be assigned to CTs. RTs either distribute the flow of work items between different tasks or join several incoming flows into one. Note that for convenience we assume 
in the above definition that all possible assignments of actors to tasks can be listed a priori. However, an extension that incorporates the concepts of "roles" is intuitively straightforward.

Definition 2: A flow process $(\mathrm{FP})$ is a triple $\mathrm{P}=\left(\mathrm{P}_{S}, \Pi, \tau\right)$ where:

- $\mathrm{P}_{\mathrm{S}}$ is a simple flow process, the parent process.

- $\Pi$ is a finite set of flow processes, the child processes.

- $\tau$ : $\mathrm{CT}_{\mathrm{P}_{\mathrm{S}}} \rightarrow \Pi$ is a function that maps CTs of $\mathrm{P}_{\mathrm{S}}$ to child processes.

A FP consists of one top-level SFP and a family of child FPs, each of which is assigned to one of the parent processes' CTs. Note that child processes need not be simple flow processes, i.e. that our model allows for multiple hierarchical levels of abstraction.

Definition 3: An instance $\vartheta_{S}: T \rightarrow \mathbb{R}^{*}$ of a simple flow process $\mathrm{P}_{\mathrm{S}}$ is a function that assigns a "productivity" $p \in \mathbb{R}$ to WTs and CTs; RTs are mapped onto "distribution vectors" $\mathbf{d} \in \mathbb{R}^{k}$, $\Sigma \mathrm{d}_{\mathrm{i}}=1$, where $\mathrm{k}$ is the number of the RT's predecessors (successors). An instance $\vartheta$ of a flow process is determined by instances of the parent process and all child processes.

The productivity of WTs and CTs measures work items per time unit to be processed. The $i$-th element of the distribution vector determines what part of the outgoing (incoming) flow is sent to (received from) the $\mathrm{i}$-th successor (predecessor) task.

Definition 4: An instance $\vartheta$ of a flow process $P=\left(P_{S}, \Pi, \tau\right)$ is co-ordinated if and only if:

a) The same productivity is assigned to neighbouring WTs and CTs of the parent process $P_{s}$. Furthermore, the ratio of input flow of the i-th predecessor (the i-th successor) of a RT with its successor (predecessor) is required to be equal to the $\mathrm{i}$-th element of the corresponding distribution vector.

b) All child processes $P_{i} \in \Pi$ are co-ordinated.

c) The productivity of each CT coincides with the productivity of every input node and every output node of its child process.

Conditions $\mathrm{a}$ and $\mathrm{b}$ require that there are neither congestions nor spare capacities at tasks and that the distribution of flow at RTs is consistent. Condition $\mathrm{c}$ implies that the productivity of a CT matches the productivities of each of the start and end nodes of the corresponding child process. In a co-ordinated FP the incoming (outgoing) flow of work items to a CT is "copied" to the start (end) nodes of the corresponding child processes. This models situations in which several actors process "simultaneously" the same flow of work items. ${ }^{1}$

Exceptions are modelled as work disruptions that cause changes in a flow process instance, i.e. they entail a decay in task productivities.

Definition 5: An exception $\sigma$ within a co-ordinated instance $\vartheta$ of a flow process $\mathrm{P}$ maps $\vartheta$ to an instance $\vartheta^{\prime}$ of $P$ such that $\vartheta^{\prime}$ is not co-ordinated and $\vartheta^{\prime}(\mathrm{t}) \leq \vartheta(\mathrm{t})$ for all WTs and CTs.

Examples of routine workflows which are suitably modelled as flow processes can be found in the banking domain. Decision-making in granting personal loans to individuals is just one of them: since personal purchase transactions by means of credit cards become more and more frequent, huge continuous flows of loan requests need to be processed by credit institutes. Cuena et al. (1990) discuss their experiences with a decision support system of this functionality for the Mexican Bancomer Bank.

Figure 1 depicts a simple prototypical flow process that leads to a decision whether to grant personal loans. Boxes in the figure indicate RTs, simple circles WTs and bold circles CTs. The black numbers inside circles show the actual productivities of the corresponding tasks; inside boxes they represent the actual distribution vectors of RTs. All this information makes up a flow process instance which states that the standard way of processing loan requests is to first generate a proposal semi-automatically by means of a decision-support sys-

\footnotetext{
${ }^{1}$ Note that the mapping of OR-nodes of the Information Control Net (ICN) formalism to flow domains results in the functionality of RTs, and that the way of assigning productivities to start and end nodes of child processes models the functionality of ICN AND-nodes.
} 
tem. This can be done by two different teams. By default, the alternative of processing requests manually is reserved for business loans. In both cases, Pérez is responsible for making the final decision whether to deny loan requests.

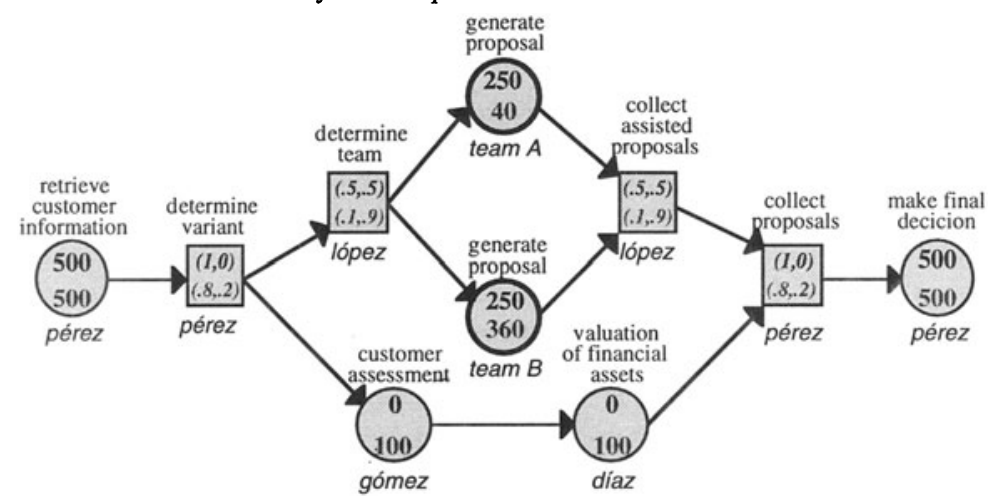

Figure 1. Dynamic co-ordination of a flow process instance for granting personal loans

\section{A SOCIAL CO-ORDINATION MECHANISM}

Case studies suggest that the actors of an organisation use to develop their own mechanisms for handling disruptions in work processes, which accomplish a satisfactory restoration of process functionality (e.g. Bowers et al., 1995). Many of these mechanisms can be characterised as lateral and social: they are based on interactions between actors with the same level of authority; there is no need to refer to process supervisors.

In this section we are concerned with the social mechanism of "helping each other out": actors are attentive to their workloads, and when one of them cannot cope with it, others assume part of the work, adapting the original workflow in an ad-hoc fashion. Drawing from Conte and Castelfranchi's (1995) theory of "Cognitive and Social Action", we present a model of the social interactions between actors in relation to the cognitive and social processes that guide them.

We first propose to consider functional co-operation as the core concept for the mechanism of "helping each other out" during the enactment of workflow processes. Subsequently, we model the process of decision-making of the involved actors. Finally, we show how sequences of social interaction arise.

\subsection{Functional co-operation in process enactment}

We set out from the assumption that actors are essentially self-motivated and autonomous: after all, their behaviour is guided by their individual goals, which they set up according to their own interests. However, as part of an organisation, actors assume the responsibility to maintain a smooth flow of work items at the tasks that have been assigned to them by process instances. In cognitive terms, they translate this responsibility into their motivation: actors responsible for WTs and CTs create the motivation of maintaining input and output flows equilibrated; responsibility for RTs leads to the motivation of distributing (recollecting) input flow(s). Actors create individual goals in order to bring situations in line with their motivations. For instance, in the loan request example team A creates the goal of achieving a pro- 
ductivity of 250 requests per hour (req/h) at the "generate proposal" task, while López creates the goal of distributing $500 \mathrm{req} / \mathrm{h}$ between team A and team B. As all process instances are initially co-ordinated, the above concepts allow for functional co-operation to arise between actors: they do not co-operate deliberately to achieve the process functionality, but are just interested in complying with their responsibilities. Nevertheless, the tasks that actors accomplish in accordance with the process model are functional with regard to the objective of the process.

When an exception occurs within a process instance the actor responsible for an affected task is in an impasse situation, as she cannot achieve her goal productivity within her individual context. So, according to her motivations, she produces a new goal of reducing input flow. This goal refers to her social context, so she asks actors to help her out of the impasse. She socially depends on these actors with respect to her goal. Obviously, in workflow processes the concept of social dependence is the inverse to producer-consumer relationships between tasks. Considering potential goals of reducing input flow for all actors of our example process instance results a network of social dependence (Sichman et al., 1994), which is shown in figure 2.

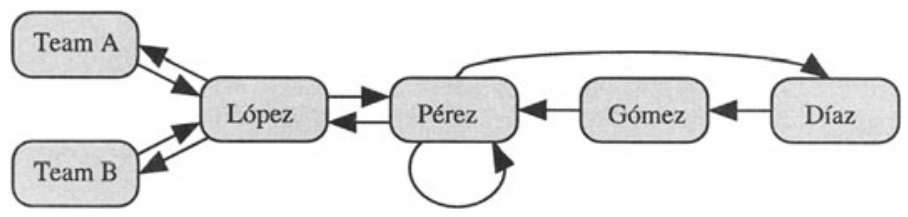

Figure 2. Network of social dependence

\subsection{Strategic and normative decision-making}

The decision of an actor whether and as to how far to comply with a request for help determines the degree to which other actors' impasses can be overcome. As on the one hand granting help implies a certain cost for the help-giver, but on the other relations of social dependence with respect to a specific goal are unilateral, there seems to be no incentive for a self-motivated actcr to co-operate (i.e. to grant help).

However, organisational processes use to remain stable over time and so do the relations of social dependence. It is likely that actors are involved repeatedly in social interactions with each other, playing different roles in different process instances. That is why actors make strategic decisions: in addition to evaluating what impact a decision has on the actual situation, they take into account its future consequences. An actor might accept a help request in order to receive help herself in an envisioned future situation, or just in order to improve her "reputation". The network in figure 2 indicates that considering just one organisational process, there are already relations of circular dependence: Díaz socially depends on Gómez who depends on Pérez who again depends on Díaz.

However, such strategic decision-making is not only quite complex but also rather brittle: models of others use to be incomplete and their behaviour need not be "perfectly rational" 2 . So, actors base their decision on prior experience, simultaneously adhering to a dynamic set of obligations, that bias these decisions in a certain way. Obligations are the result of the application of norms to a specific situation:

\footnotetext{
${ }^{2}$ We use the term in the intuitive sense here. Although game theory provides a definition on what it means to act (individually) rational in a single encounter, there is no general theory for repeated interactions.
} 
- Social norms demand certain behaviours towards actors. Either they create delayed obligations, as the "norm of reciprocity" which indicates that a help-receiver "owes a favour" to the help-giver, or they oblige an actor to reconsider an actual decision. An example of the latter case is the "norm of politeness" which states that before increasing the output flow at a task, actors are obliged to ask the affected actors for approval. It is of mayor importance for workflow processes as it motivates forward propagation of "help requests".

- Process norms create obligations that refer to tasks. A process norm might indicate that individual loans should be processed semi-automatically by using decision-support systems instead of manually by applying the procedure for checking business loans.

The function of norms is to incline actors' decisions towards a ratio of acceptance of help requests which goes beyond mere strategic self-interest: while denying help to an individual actor just implies the risk that the latter withholds future co-operation, not complying with obligations may result in retaliation from all actors that become aware of that norm transgression.

\subsection{Social interaction}

The decision whether to grant help in a single social interaction is based on an actor's estimate of the "cost" of acceptance. In our highly constrained model of workflow processes, this cost is primarily determined by the behaviour of the actors that the potential help-giver is socially dependent on. So, she might propagate the request backward, giving rise to a sequence of social interactions. Actors of RTs may fulfil a request for help just by redistributing flow. However, the "norm of politeness" obliges them to ask the affected actor for approval, which leads to a forward propagation of requests.

In the sequel we outline one possible sequence of social interactions in the loan request example of figure 1, in which all actors behave "conservatively" (i.e. they ask actors they are dependent on before deciding about a help-request) and abide to norms. Suppose an exception occurs at the "generate proposal" task, and that team A asks López to reduce the input flow by $250 \mathrm{req} / \mathrm{h}$, i.e. to "retire" that path of the process instance entirely. López reasons that her acceptance would creates an impasse to her, with respect to which she is socially dependent on Pérez. However, she prefers to redistribute flow. Fulfilling her obligation, she asks team B if they are willing to accept an additional flow of $250 \mathrm{req} / \mathrm{h}$. Team B replies that they only admit $110 \mathrm{req} / \mathrm{h}$. As a consequence, López asks Pérez to reduce her output flow by the remaining $140 \mathrm{req} / \mathrm{h}$. The norm of politeness urges Pérez to ask Gómez if she agrees to augment her input flow by $140 \mathrm{req} / \mathrm{h}$. The request keeps on propagating, until Gómez finally comes up with her acceptance of reducing $100 \mathrm{req} / \mathrm{h}$. López passes this answer back to team A, which finally needs to cope with a flow of $40 \mathrm{req} / \mathrm{h}$. The grey numbers in figure 1 indicate the result of the above interactions.

Note that not every sequence of social interactions leads to a co-ordinated flow process. In such cases impasses are "propagated" through the process instance. If this does not lead to coordination within a certain amount of time (i.e. an impasse "oscillates") exception management needs to be delegated to a process supervisor.

\section{CO-ORDINATION SUPPORT}

Efficient computer support requires cognitive modelling of actors. In workflow processes actors must possess knowledge concerning how to perform the tasks that they are responsible for. However, as we aim to support the dynamic re-coordination of process instances by 
means of a social mechanism, we are primarily interested in the types of social knowledge that an actor needs to be endowed with. We claim that actors need social knowledge about

- social dependence relations which enables them to find potential addressees for help requests. Furthermore, by reasoning with the network of social dependence the "importance" of a certain actor can be determined and the potential cost of rejecting her help request can be estimated;

- norms which allows them to deduce personal and foreign obligations, that may influence the cost of present and future behaviour;

- models of others which include models concerning general dispositions and social strengths of other actors. This knowledge can be augmented dynamically by "learning" about actual obligations and attitudes of others;

- social interactions which describes the potential structures of conversations between actors along with which sequences of social interactions occur. It specifies how actors may respond to speech acts in a meaningful way.

In addition to this, an actor is endowed with a social reasoning mechanism. This mechanism guides her decision-making in social interactions by comparing the cost of acceptance of a help request with the cost of its rejection and of potential obligation violations.

As we approach workflow management with the goal of rendering co-ordination assistance rather than providing information for accountability (Bowers et al., 1995), we favour a distributed architecture for systems intended to encourage lateral co-ordination: actors will only accept such a system if they can be sure that their own strategic information remains private. This is accounted for by keeping this information physically separated, together with its "owner". So, we provide each actor with its personal Social Information Agent (SIA).

SIAs provide communication services for actors and support operation and update of the actors' social knowledge. They are provided with "static" information derivable from process instances (i.e. networks of social dependence) as well as with knowledge concerning conversation structures and mutually agreed norms. Furthermore, they dynamically gather historical information about process enactment and social interactions. On this basis, SIAs

- provide contextual information for social interactions by tracking conversation states and by retrieving social dependence relations,

- update models of the attitudes of others on the basis of historical information, and present them along meaningful variables (degree of acceptance, degree of norm transgression),

- keep track of personal and foreign obligations (e.g. indicating who owes a favour to whom) and may notify potential obligation violations.

Finally, it is worth pointing out that SIAs and traditional workflow approaches are not exclusive but orthogonal: While SIAs focus on co-ordination aspects of process enactment, a system with a global view can be used to handle mayor errors and to gather process data for accountability and process redesign.

\section{DISCUSSION}

In this paper we have presented a formalism for modelling workflows as flow processes and proposed a social mechanism that achieves lateral re-coordination of process instances in exception situations. By acknowledging repairs in a default workflow, which are less optimal than those achieved by anticipative approaches, this mechanism provides the potential for more flexible and less prescriptive exception management.

In the frame of a computational validation of our model of co-ordination support we are currently working on an executable model of a social actor. The core of this model resides in 
a social reasoning functionality, that integrates strategic and normative aspects of decisionmaking. Such an agent will be able to act independently within our co-ordination framework, making up what Decker and Lesser (1995) call an "mixed human and computational agent system". On this basis dynamic co-ordination can be achieved for workflow processes that comprise manual and automated tasks.

\section{REFERENCES}

Bowers, J.; Button, G. and Sharrok, W. (1995) Workflow from Within and Without, in Proc. 4th Europ. Conf. on CSCW, Kluwer Academic Publishers

Conte, R. and Castelfranchi C. (1995) Cognitive and Social Action, UCL Press

Cuena, J.; García-Serrano, A. and Marquez, J. (1990) CYRCE: A Pattern of Knowledge Representation and Knowledge Acquisition for Decisions on Personal Loans. Expert Systems With Applications, 1, 155-160

Decker, K. and Lesser, V. (1995) Coordination Assistance for Mixed Human and Computational Agent Systems. UMass CS Tech. Rep. 95-31, 1995

Eder, J. and Liebhart, W. (1996) Workflow Recovery, lst Intl. Conf. on Cooperative Information Systems, IEEE Computer Society Press

Malone, T. and Crowston, K. (1994) The Interdisciplinary Study of Coordination. ACM Computing Surveys, 26, 87-119

Nutt, G. (1993) Using Workflow in Contemporary Applications. Univ. of Colorado Report CU-CS-663-93

Ossowski, S.; García-Serrano, A. and Cuena, J. (1996) Emergent Co-ordination of Flow Control Actions Through Functional Co-operation of Social Agents, in Proc. 12th Europ. Conf. on Artificial Intelligence, John Wiley and Sons

Robinson, M. (1993) Design for Unanticipated Use, in Proc. 3rd Europ. Conf. on CSCW, Kluwer Academic Publishers

Sichman, J.; Demazeau, Y.; Conte, R. and Castelfranchi, C. (1994) A Social Reasoning Mechanism Based On Dependence Networks, in Proc. 11th Europ. Conf. on Artificial Intelligence, John Wiley and Sons

Singh, M. and Huhns M. (1994) Automating Workflows for Service Order Processing: Integrating $\mathrm{AI}$ and Database Technologies. IEEE Expert, 10, 19-23

\section{BIOGRAPHY}

Ana García-Serrano received the M.Sc. degree in Mathematics from Complutense University of Madrid in 1982. In 1987 she obtained the Ph.D. degree in Computer Science from the AI Department of Technical University of Madrid, where she is currently teaching Computational Logic and AI as an Associate Professor. Her research interests include Distributed AI, Computer Supported Co-operative Work and Natural Language Processing.

Sascha Ossowski received the M.Sc. degree in Computer Science from Oldenburg University (Germany) in 1993. He is currently working as a research associate in the AI Department of Technical University of Madrid, where he is preparing a Ph.D. thesis in the area of Distributed AI and Co-ordination Theory. He is holding an HCM research training fellowship of the European Union. 